\title{
Effects of Oxygen-Induced Lung Damage on Megakaryocytopoiesis and Platelet Homeostasis in a Rat Model
}

\author{
JIE YANG, MO YANG, FENG XU, KAREN LI, SIMON K.M. LEE, PAK-CHEUNG NG, \\ JANET S.K. TAM, PATRICK M.P. YUEN, AND TAI-FAI FOK \\ Department of Paediatrics (J.Y., M.Y., F.X., K.L., P-C.N., P.M.P.Y., T-F.F.), Lee Hysen Clinical Research \\ Laboratories (S.K.M.L.), Department of Anatomical and Cellular Pathology (J.S.K.T.), Prince of Wales \\ Hospital, The Chinese University of Hong Kong, Shatin, Hong Kong, China
}

\begin{abstract}
The association between lung injury and thrombocytopenia was investigated by comparing the megakaryocyte and platelet counts, and platelet activation using P-selectin as a marker, between the prepulmonary (right atrial) and postpulmonary (left atrial) blood in adult and neonatal (preterm and term) rats with and without hyperoxic lung injury. In the healthy controls, the postpulmonary blood had lower megakaryocyte count (prepulmonary versus postpulmonary: Preterm: $8.7[0.6]$ versus $3.9[0.3]$ per ml, $p<0.001$; Term: 8.7[1.1] versus 2.6[0.4] per ml, $p<$ 0.001; Adult: median [interquartile ranges]: $2.5[1.0,5.0]$ versus $1.0[0,3.0]$ per ml, $p<0.001$ ), higher platelet count (prepulmonary versus postpulmonary: Preterm: $491.2[11.1] \times 10^{9} / \mathrm{L}$ versus 595.1[10.2] $\times 10^{9} / \mathrm{L}, p<0.001$; Term: 472.5[19.9] $\times 10^{9} / \mathrm{L}$ versus 579.3[26.2] $\times 10^{9} / \mathrm{L}, p<0.001$; Adult: $513.9[31.5] \times$ $10^{9} / \mathrm{L}$ versus $\left.664.7[28.8] \times 10^{9} / \mathrm{L}, p<0.001\right)$, but similar $\mathrm{P}$-selectin expression. In contrast, the lung-damaged animals did not show any such differences in either megakaryocyte or platelet count, but P-selectin expression was greater in the postpulmonary blood (prepulmonary versus postpulmonary: Preterm: 38.7[3.9] versus 56.4[4.9]\% platelets, $p=0.02$; Term: 40.9[2.0] versus $54.0[4.2] \%$ platelets, $p=0.002$; Adults: 30.0 [3.6] versus $49.1[4.7] \%$ platelets, $p=0.003)$. Peripheral platelet and intra-
\end{abstract}

ABSTRACT

pulmonary megakaryocyte counts in the lung-damaged rats were significantly lower than those in their respective controls. Intrapulmonary thrombi or platelet aggregation were detected in the lung-damaged rats but not in the controls. These findings showed that hyperoxic lung damage reduced circulating platelets through (1) failure of the lungs to retain and fragment megakaryocytes to release platelets, and (2) platelet activation leading to platelet aggregation, thrombi formation and platelet consumption. The magnitude of platelet reduction was physiologically significant, as demonstrated by higher counts of megakaryocyte colony forming units in the bone marrow culture of the animals in the hyperoxia group when compared with the controls. (Pediatr Res 54: 344-352, 2003)
Abbreviations
AchE, acetylcholine esterase
CFU-MK, colony forming unit of megakaryocyte
FITC, fluorescin isothiocyanate
HPF, high power field
IgG, IgG
MK, megakaryocyte
PE, phycoerythrin

Thrombocytopenia is a common complication in neonates and may affect up to $7.6 \%$ of all newborns $(1,2)$. The condition is particularly prevalent in sick infants. In a survey by Castle et al. $58 \%$ and $22 \%$ of infants receiving intensive care had platelet count $<150 \times 10^{9} / \mathrm{L}$ and $100 \times 10^{9} / \mathrm{L}$, respectively (3). Congenital or early onset ( $<72 \mathrm{~h}$ of birth) neonatal thrombocytopenia may be caused by immunologic causes including isoimmune or maternal autoimmune thrombocytopenia, failure

Received August 29, 2002; accepted January 28, 2003.

Correspondence: Dr. Tai-fai Fok, Department of Paediatrics, Prince of Wales Hospital, Shatin, N.T., Hong Kong; e-mail: taifaifok@cuhk.edu.hk

This study was supported by an Earmarked Grant of the Research Grant Council, Hong Kong.

DOI: 10.1203/01.PDR.0000079186.86219.29 of platelet production such as that in the thrombocytopeniaabsent-radii syndrome, congenital infection, and bone marrow suppression due to maternal hypertension, preeclampsia, and diabetes mellitus $(1,4)$. The etiology however remains unclear in a large proportion of cases $(3,5,6)$. The late onset $(>72 \mathrm{~h}$ of birth) type of thrombocytopenia is often attributed to sepsis (7), although an infective cause is often not identifiable in the affected infants. Previous workers have observed that this category of thrombocytopenia is particularly common in newborns with lung disease such as respiratory distress syndrome $(5,8-11)$, and the degree of platelet reduction is directly related to the severity of the underlying lung disease, the concentration of inspired oxygen $(9,11)$, and the ventilation pressure $(8,11)$. In a study on rabbits, mechanical ventilation 
was associated with a mean decrease of platelet count by $37 \%$ (8). In human adults, a reduction in platelet survival time has also been observed in patients with chronic obstructive airway disease and pulmonary hypertension (12). These observations strongly suggest the presence of an association between lung damage and platelet homeostasis.

Circulating platelets are released from fragmentation of megakaryocytes in the capillary blood vessels $(13,14)$. While the fetal liver capillary sinusoids $(15)$ and the placenta $(16,17)$ are possible sites of megakaryocyte (MK) fragmentation during the early and late fetal life respectively, it is likely that during postnatal life, MK fragmentation takes place mainly in the lung, the first capillary bed encountered by the cells leaving the bone marrow $(14,18,19)$. The presence of MK cells in the human lungs was described by Aschoff over 100 years ago (20). Subsequently, a number of studies have demonstrated that MK were greater in abundance and cytoplasmic volume in the central venous (prepulmonary) blood than in the arterial (postpulmonary) blood (21-25), providing an indirect evidence that these cells are fragmented in the lungs. Studies on patients on cardiopulmonary bypass have shown that the lungs are net removers of MK from the circulation (25). Using a mathematical model based on the quantity of MK plasma volume reduction and platelet released in the postpulmonary circulation, Trowbridge et al. have provided evidence that all circulating platelets are produced in the pulmonary circulation (26). More recently, the importance of the lung in platelet homeostasis is confirmed by the direct observation under electron microscopy the release of platelets from MK cells in the pulmonary capillary bed (13).

While the lung might play a key role in platelet formation, its extensive capillary bed might also be responsible for platelet entrapment and consumption in infants who have sustained lung injury (10). Endothelial damage, commonly observed in the lungs of infants with respiratory distress syndrome, barotrauma, or oxygen-induced lung damage, is associated with thrombi formation, which consists of inflammatory cells, fibrin debris, proteinaceous exudates, and platelet aggregates. We postulate that in infants with injured lungs, a reduction in peripheral platelet count is caused by the dual process of impaired platelet formation and increased platelet consumption in the lungs. The present study was carried out to evaluate this hypothesis in neonatal (full-term and preterm) and adult rat models. The animals suffered from lung injury resulting from exposure to high concentration oxygen. This method of inflicting lung injury is cheap, convenient, and reproducible, and mimics the oxygen-induced lung injury commonly seen in newborns receiving intensive care.

\section{METHODS}

Animal models. Preterm Sprague-Dawley rats delivered by hysterotomy at 21-d gestation, and full-term rats delivered vaginally at $22-\mathrm{d}$ gestation were by random assignment exposed to $>95 \%$ oxygen or room air for $7 \mathrm{~d}$ as previously described (27-29). Adult rats were similarly exposed for $60 \mathrm{~h}$. Previous studies have shown that both term and preterm neonatal rats would sustain significant acute lung injury resulting in alveolar hemorrhage, interstitial edema, and damage of the endothelium and lung parenchyma after prolonged exposure to high concentration oxygen. The adult rats were exposed to hyperoxia for a shorter duration because of their intolerance to hyperoxia and high mortality after $72 \mathrm{~h}$ of hyperoxic exposure (30).

At the end of exposure, the animals in the hyperoxic groups were noticeably thriving less well than the controls. They, however, remained active and their general condition appeared to be stable. From each of the hyperoxia and room-air groups, 26 preterm pups and 30 full-term pups, each from a separate litter, and 18 adult rats were randomly selected for the following studies: peripheral platelet count, lung histology, lung MK count, and bone marrow MK progenitors assay. The same adult rats were also used for the determination of prepulmonary and postpulmonary MK and platelet counts, and P-selectin levels. However, because of their small blood volume, separate groups of term and preterm neonatal rats $(n=10$ from each of hyperoxia and control groups for both) were used for the study of the prepulmonary and postpulmonary blood. The animal was anesthetized with intra-peritoneal ketamine $(90 \mathrm{mg} / \mathrm{kg})$. Peripheral platelet count was estimated in EDTA blood samples collected by snipping the tail of the adult rat, and by needle aspiration of the left atrium of the preterm or term neonatal rat immediately after its thorax was opened to expose the heart. Lung and bone specimens were obtained for lung histology and bone marrow studies described below. For the estimation of prepulmonary and postpulmonary MK and platelet counts and P-selectin, EDTA blood samples $(2.0 \mathrm{~mL}$ each $)$ were withdrawn simultaneously from the right and left atrium by direct puncture immediately after the thorax was opened to expose the heart. All the animals were killed with intraperitoneal pentobarbital after completion of sample collection. The study was approved by the Committee on Research Animal Welfare of the Institution.

Lung histology and thrombi formation. The animals' lungs were fixed with $10 \%$ buffered formalin at a constant inflation pressure of $20 \mathrm{~cm} \mathrm{H}_{2} \mathrm{O}$ as previously described (31). Lung sections were obtained from identical locations at the left lung base and the right middle and lower lobes. Histologic examination for evidence of lung damage and thrombi formation was carried out under light microscopy ( $4 \mu \mathrm{m}$ paraffin hematoxylineosin sections), and when necessary, also under electron microscopy $(80 \mathrm{~nm}$ cut, stained with uranyl acetate and lead citrate) (13) using a Philips CM100 electron microscope (Philips Ltd., Netherlands).

Lung MK identification. Frozen sections $(5 \mu \mathrm{m})$ were labeled with acetylcholine esterase (AchE) (Sigma Chemical Co., Saint Louis, U.S.A.) and counterstained with hematoxylin to identify MK as previously described (32). In brief, the slide was fixed with $1 \%$ paraformaldehyde in $0.1 \mathrm{M}$ PBS (pH 6.0), washed with $0.1 \mathrm{M}$ PBS, and stained with AChE solution for $4 \mathrm{~h}$ at room temperature. The slide was then counterstained with Harris Hematoxylin for 1-5 min., rinsed with water, treated with a weak ammonia solution for $1 \mathrm{~min}$., and then washed in water several times. Lung MK were counted in 20 random fields from three lung sections per animal at $40 \times$ magnification. The fields with large bronchi or vessels were excluded. 
Circulating megakaryocytes and platelets. Blood platelets were quantified under phase-contrast microscopy (Nikon Eclipse TE300, Japan) after red cells were lysed with $1 \%$ ammonium oxalate. Circulating MK count was estimated as previously described (33). In short, $3 \mathrm{~mL}$ of EDTA blood were passed through an assembled syringe filter (Millipore, Herfordshire, Ireland) containing a nucleopore polycarbonate membrane of $5 \mu \mathrm{m}$ pore diameter (18) followed by two washes each with $2 \mathrm{~mL}$ saline. After drying, the membrane was stained with AchE for MK identification as described above.

$P$-selectin expression on platelets. P-selectin (CD62P) expression on platelets, which is an indication of platelet activation, was estimated by flow cytometry (FACSCalibur, BectonDickinson, San Jose, CA, U.S.A.) and indirect immunostaining (34). Platelets were identified with phycoerythrin (PE)-labeled anti-CD61 and P-selectin positive platelets were identified with polyclonal rabbit anti-CD62P IgG detected with a secondary fluorescein (FITC)-conjugated anti-rabbit IgG. The control tubes contained FITC-anti-rabbit IgG and PE-conjugated hamster IgG or FITC-anti-rabbit IgG and PE-anti-CD61. All antibodies were purchased from Pharmingen, San Diego, CA, U.S.A. Twenty thousand CD61 positive events were acquired for each analysis. The percentage of platelets expressing CD62P was obtained by subtracting the background signals of the nonimmune antibody control $(2.50 \pm 1.00 \%$ of CD61 positive cells).

Bone marrow megakaryocytic progenitor assay. Bone marrow cells harvested from the femurs were cultured using the plasma-clot culture method (35). Colony forming unit of megakaryocyte (CFU-MK) was identified by AchE and hematoxylin staining as described above. A CFU-MK was defined as a cluster of 3 or more AchE positive cells (32).

Statistical analysis. Between-group comparisons were performed using 2-tailed Student $t$ test or Mann Whitney Rank Sum test, where appropriate. Prepulmonary and postpulmonary comparisons were made using the paired $t$ test or Wilcoxon Sign Rank test. A p value $\leq 0.05$ was considered statistically significant. All values were expressed as mean [SEM] unless otherwise stated.

\section{RESULTS}

All the values given in this section are in mean [SEM] except when the values are not normally distributed, in which case they will be expressed as median [interquartile range].

Peripheral blood platelet count. In the preterm neonatal ( $n$ $=26$ in each of hyperoxia and control groups), term neonatal ( $n=30$ in each of hyperoxia and control groups) and adult ( $n$ $=18$ in each of hyperoxia and control groups) animals, total peripheral platelet count was significantly lower in the oxygentreated groups than in the respective controls (Fig. 1) (Control versus hyperoxia group: Preterm: $443.6[17.3] \times 10^{9} / \mathrm{L}$ versus $260.0[17.4] \times 10^{9} / \mathrm{L}, p<0.001$; Term: $461.9[15.2] \times 10^{9} / \mathrm{L}$ versus $189.1[9.0] \times 10^{9} / \mathrm{L}, p<0.001$; Adult: $653.8[39.0] \times$ $10^{9} / \mathrm{L}$ versus $\left.443.6[16.2] \times 10^{9} / \mathrm{L}, p<0.001\right)$

Lung morphology, thrombi formation, and lung MK. Features of lung injury consisting of patchy areas of intra-alveolar hemorrhage, interstitial edema, and disruption of the alveolar

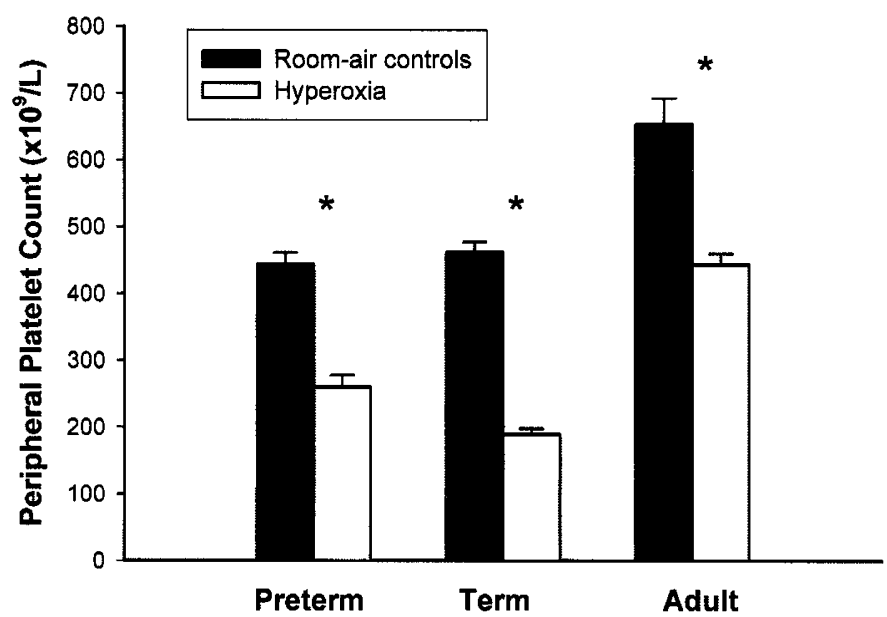

Figure 1. Peripheral platelet counts of the preterm neonatal, term neonatal, and adult rats $(n=26,30$, and 18 , respectively, in each of hyperoxia and control groups). In all three groups, the animals with hyperoxic lung damage had significantly lower platelet counts than their respective controls. Values are expressed as mean (SEM). ${ }^{*} p<0.001$

structure were present in all the oxygen treated animals. In contrast, all the room-air controls had normal lung morphology. (Fig. 2A,B) Extensive thrombi formation was observed in the injured parts of the lungs in all the preterm and full-term neonatal rats (Fig. 2C,D). In the adult rats, vascular thrombi were not observed under light microscopy despite severe lung damage, but examination under electron microscopy demonstrated extensive platelet aggregation and adhesion to the capillary endothelium (Fig. 2E,F). Thrombi formation or platelet aggregation was not detected in the lungs of any of the room-air controls.

The number of MK was estimated in 20 randomly selected high-power fields of the lung sections of each of the animals (Fig. 3). In the preterm neonatal, term neonatal, and adult $(n=$ 26,30 , and 18 , respectively, in each of hyperoxia and control groups) rats, the hyperoxia groups had significantly lower counts than those in the room-air controls (Preterm: control versus hyperoxia group: 19.7[1.4]/20 HPF versus 5.0[1.2]/20 HPF, $p<0.001$; Term: 20.9[1.8]/20 HPF versus $4.8[0.6] / 20$ HPF, $p<0.001$; Adult: 9.9[0.9]/20 HPF versus 6.3[0.6]/20 HPF, $p=0.002$ ).

Prepulmonary and postpulmonary MK and platelet counts. Figure 4 illustrates the morphology of an MK cell observed in the peripheral blood. Among the room-air controls in all the preterm neonatal $(n=10)$, term neonatal $(n=10)$, and adult ( $n=18)$ groups, MK count was significantly higher in the prepulmonary (right atrial) than in the postpulmonary (left atrial) blood (Fig. 5A) (prepulmonary versus postpulmonary: Preterm: 8.7[0.6] versus 3.9[0.3] per $\mathrm{ml}, p<0.001$; Term: 8.7[1.1] versus 2.6[0.4] per ml, $p<0.001$; Adult: median [interquartile ranges]: $2.5[1.0,5.0]$ versus $1.0[0,3.0]$ per $\mathrm{ml}$, $p<0.001)$. The relationship for platelet count was reversed, being significantly higher in the postpulmonary blood (Fig. $5 B$ ) (pre- versus post-: Preterm: $491.2[11.1] \times 10^{9} / \mathrm{L}$ versus $595.1[10.2] \times 10^{9} / \mathrm{L}, p<0.001$; Term: $472.5[19.9] \times 10^{9} / \mathrm{L}$ versus $579.3[26.2] \times 10^{9} / \mathrm{L}, p<0.001$; Adult: $513.9[31.5]$ $\times 10^{9} / \mathrm{L}$ versus $\left.664.7[28.8] \times 10^{9} / \mathrm{L}, p<0.001\right)$. These 

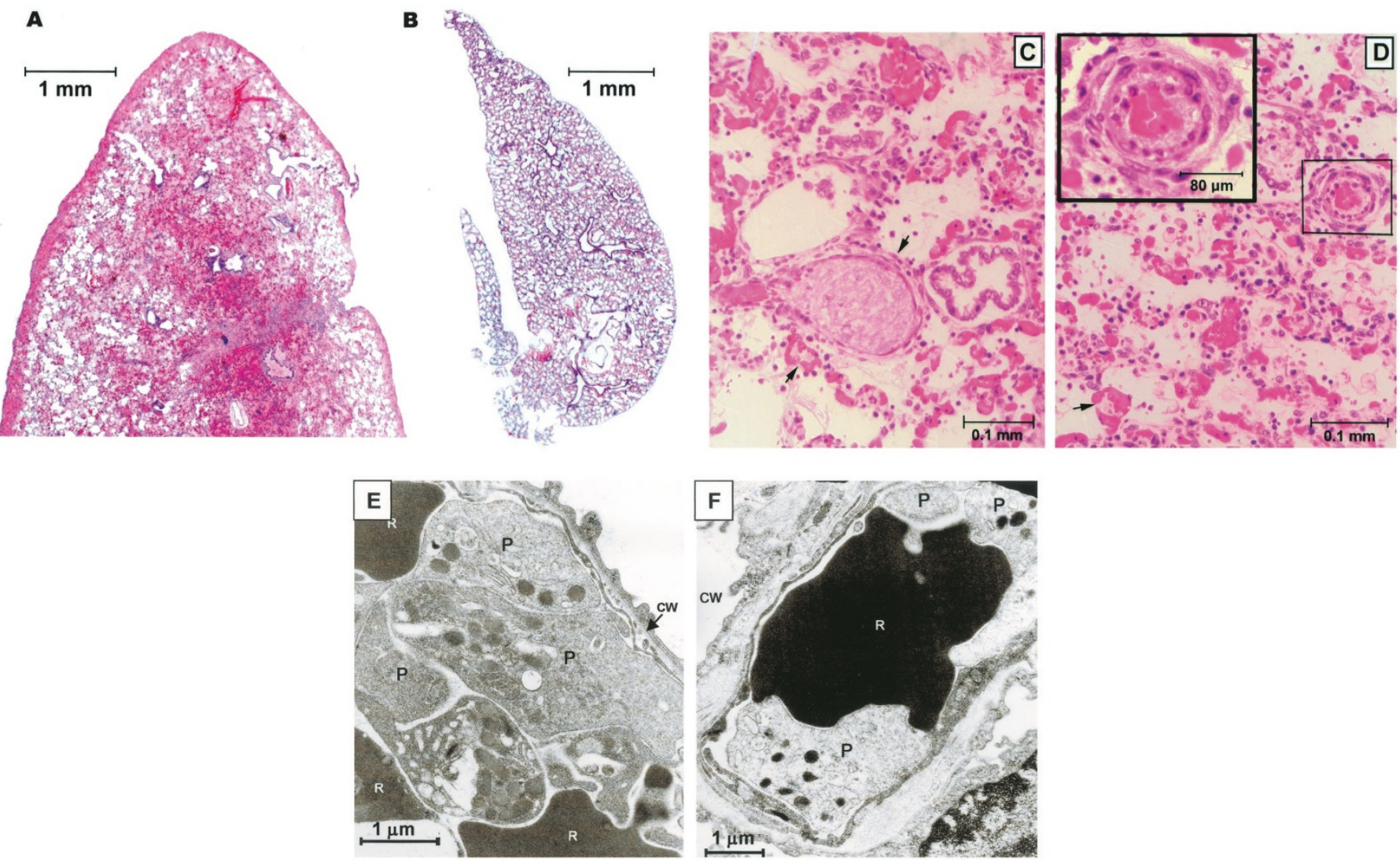

Figure 2. (A): A representative low power lung section obtained from a term neonatal rat in the hyperoxic group, showing extensive damage of the alveolar architecture, infiltration by inflammatory cells, and hemorrhage into the alveolar and interstitial space. A lung section from a control term neonatal rat $(B)$ shows normal lung morphology. Similar observations are made in the lung sections obtained from preterm neonatal and adult rats in each of the hyperoxia and control groups (not shown). (C) A high power section obtained from the injured part of a preterm neonatal rat showing extensive lung damage with hemorrhage and thrombi formation. A large intravascular thrombus is present in the center of the photomicrograph (indicated by arrow). (D) A similar section obtained from the injured part of the lung of a term neonatal rat showing similar changes. The inset shows the presence of an intravascular thrombus. $(E)$ and $(F)$ : Electron micrographs of the lung of a lung-damaged adult rat showing platelet $(\mathrm{P})$ aggregation and adhesion to the endothelial wall of the pulmonary capillary blood vessels $(\mathrm{CW})$. The platelets are deformed, suggesting that they have been activated. $\mathrm{R}=$ red blood cell.

differences were not observed in the oxygen-treated rats in which the prepulmonary and postpulmonary levels were similar in both MK (Fig. $5 A$ ) (pre- versus post-: Preterm [n $=10]: 7.8[0.7]$ versus $7.4[0.8]$ per $\mathrm{ml}, p=0.39$; Term $[\mathrm{n}=$ 10]: 7.4[0.7] versus 6.3[0.5] per ml, $p=0.13$; Adult $[\mathrm{n}=$ 18]: median [interquartile ranges]: $2.0[1.0,3.0]$ versus $2.0[1.0,3.0]$ per ml, $p=0.125$ ) and platelet (Fig. $5 B$ ) counts (pre- versus post-: Preterm $[\mathrm{n}=10]: 312.9[18.1] \times 10^{9} / \mathrm{L}$ versus $291.7[16.8] \times 10^{9} / \mathrm{L}, p=0.230$; Term $[\mathrm{n}=10]$ : $312.9[17.4] \times 10^{9} / \mathrm{L}$ versus $308.9[20.3] \times 10^{9} / \mathrm{L}, p=0.7$; Adult $[\mathrm{n}=18]: 426.8[14.4] \times 10^{9} / \mathrm{L}$ versus $424.3[14.9] \times$ $\left.10^{9} / \mathrm{L}, p=0.851\right)$. In all three groups, the differences between the prepulmonary and postpulmonary values were significantly greater in the room-air controls than in the hyperoxia rats for both MK (Preterm: 53.6[5.2] versus 4.5[5.2]\%, $p<0.001$; Term: $68.7[4.5]$ versus $-10.3[7.4] \%$, $p<0.001$; Adults: 64.6[8.5] versus 18.5[9.0], $p<0.001$ ) and platelet (Preterm: 21.7[3.4] versus -5.7[4.5]\%, $p<$ 0.001; Term: 23.6[5.9] versus $-1.1[3.4] \%, p=0.002$; Adult: 34.1[7.1] versus 0.2[3.3], $p<0.001$ ).

Prepulmonary and postpulmonary P-selectin expression. The estimation of P-selectin expression of platelets in the right and left atrial blood allowed the determination of the propor-

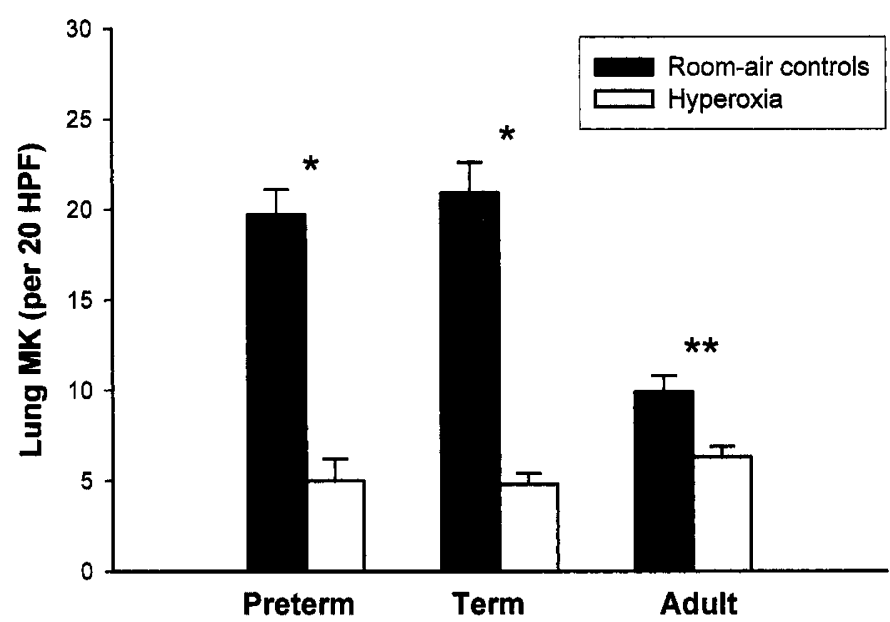

Figure 3. The quantity of megakaryocytes (MK) present in 20 randomly selected high-power fields of each of the animals in the preterm neonatal, term neonatal, and adult ( $n=26,30$, and 18 , respectively, in each of hyperoxia and control groups) groups with and without hyperoxic lung damage. In all three groups, the lung MK counts in the animals with hyperoxic lung damage were significantly lower than those in their respective room-air controls. Values are expressed as mean (SEM). ${ }^{*} p<0.001,{ }^{*} p=0.002$. 


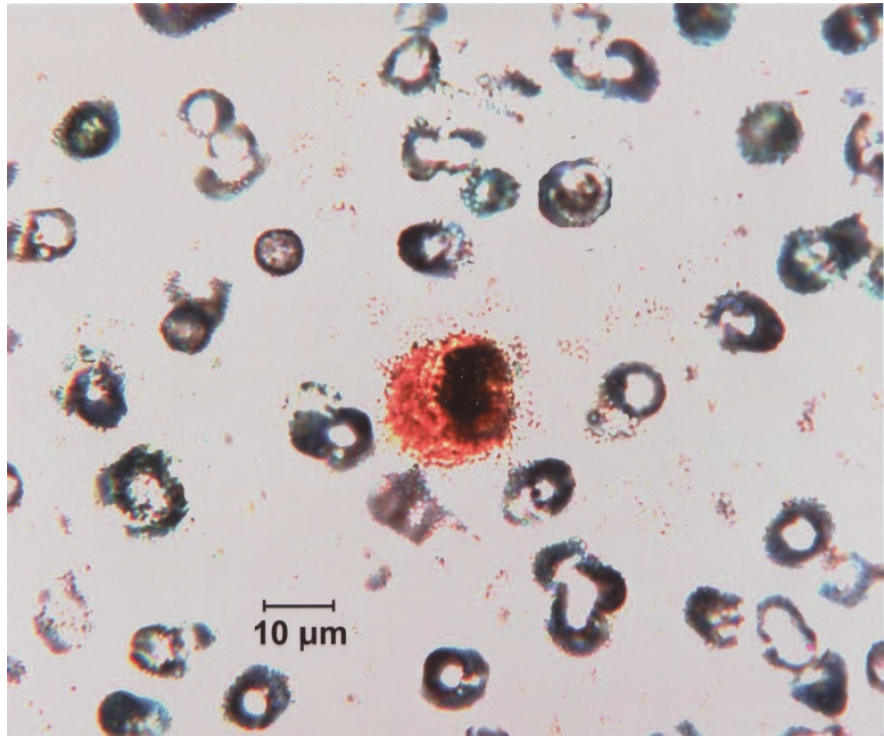

Figure 4. An MK identified by AchE staining after prepulmonary or postpulmonary blood was passed through an assembled syringe filter containing a nucleopore polycarbonate membrane of $5 \mu \mathrm{m}$ pore diameter.

tion of activated platelets in the prepulmonary and postpulmonary circulation, respectively. Figure 6 shows the percentage of platelets with P-selectin expression in the prepulmonary and postpulmonary blood in the hyperoxia and room-air control rats. In the controls, there was no significant difference in CD62P expression between the prepulmonary and postpulmonary blood (pre- versus post-: Preterm $[n=10]$ : 18.3[1.5] versus $15.5[2.3] \%$ platelets, $p=0.22$; Term $[n=10]$ : 18.6[1.6] versus $16.5[1.6] \%$ platelets, $p=0.32$; Adults $[n=$ 18]: 10.4[1.4] versus 12.8[2.6] \% platelets, $p=0.347$ ). In contrast, in the hyperoxia group, P-selectin expression was significantly higher in the postpulmonary blood than in the prepulmonary blood (pre- versus post-: Preterm $[\mathrm{n}=10]$ : 38.7[3.9] versus 56.4[4.9] \% platelets, $p=0.02$; Term $[\mathrm{n}=$ 10]: 40.9[2.0] versus 54.0[4.2] \% platelets, $p=0.002$; Adults $[n=18]: 30.0[3.6]$ versus 49.1[4.7] \% platelets, $p=0.003$ ). The difference between the prepulmonary and postpulmonary values was significantly greater in the hyperoxia rats than in the controls (Preterm: 62.3[24.7] versus - 15.5[11.2] \%, $p=$ 0.009; Term: 31.8[7.1] versus 7.5[10.1] $\%, p=0.008$; Adult: $92.9[27.6]$ versus 22.2[17.4], $p=0.004$ ). Comparing the baseline (prepulmonary) and postpulmonary P-selectin expression between the two groups, both parameters in the lung-damaged animals were significantly greater than their respective control groups ( $p<0.001$ in all three groups).

Bone marrow CFU-MK. In both the neonatal (preterm $[n=$ 26 in each of hyperoxia and control groups] and full-term $[\mathrm{n}=30$ in each of hyperoxia and control groups ]) and adults [ $n=18$ in each of hyperoxia and control groups] rats, the bone marrow CFU-MK was significantly higher in the hyperoxia groups than in the room-air controls (control versus hyperoxia: Preterm: 13.2[1.3] versus $24.5[2.4]$ per $2 \times 10^{5}$ cells, $p<0.001$; Term: $10.9[0.7]$ versus $16.8[1.0]$ per $2 \times 10^{5}$ cells, $p<0.001$; Adults: $21.7[1.8]$ versus $31.3[2.9]$ per $2 \times 10^{5}$ cells, $p=0.008$ ) (Fig. 7).

\section{DISCUSSION}

In this study, we investigated the effect of hyperoxic lung injury on megakaryocyte and platelet homeostasis by examining the changes in the MK and platelet counts in the blood before and after passage through the lung. In the healthy adult rats, the MK content in the prepulmonary circulation was similar to that reported by Pederson et al. (24) To the best of our knowledge, the circulating MK count in neonatal rats has not been previously reported. Our findings showed that it was more than thrice the adult values in both the preterm and term newborn pups. In the healthy adult animals, passage of mixed venous blood through the pulmonary capillary bed resulted in a significant reduction in $\mathrm{MK}$ count by $65 \%$, which was associated with a $34 \%$ increase in platelet count. These findings were consistent with those previously reported by other investigators $(21,23,24)$, and provided further evidence that the lung is an important site for MK fragmentation and platelet release. A similar albeit slightly lesser prepulmonary and postpulmonary gradient in MK and platelet counts was also observed in the term and preterm neonatal pups, thus confirming the role of the lung in platelet release in the newborns. In the animals with hyperoxic lung damage, the MK and platelet counts in the postpulmonary circulation were almost identical to those in the prepulmonary blood, indicating that there was no significant MK consumption or net platelet production in the pulmonary capillary bed. These findings supported our hypothesis that the process of MK fragmentation to release platelets was impaired in the damaged lung. On histologic examination, the lung tissues of both the adult and neonatal (term and preterm) animals in the hyperoxia group contained significantly fewer MK cells when compared with the room-air controls. Lung MK counts in the hyperoxia groups could have been affected by the presence of lung injury, which might impair lung inflation and increase the amount of lung tissues in a given microscopic field, thus resulting in a falsely high MK count. We have tried to minimize this confounding effect by fixing the lungs under a constant inflation pressure of $20 \mathrm{~cm}$ $\mathrm{H}_{2} \mathrm{O}$. Furthermore, since under-inflation would only overestimate lung MK count, the artifact would not affect our conclusion that hyperoxic lung injury was associated with a decrease in lung MK. The significance of this association is not clear. It could be interpreted as the result of more efficient consumption of MK to produce platelets in the hyperoxia group. However, if this was indeed the case, the reduction in lung MK should have been accompanied by more complete clearance of MK from the pulmonary blood, and a drop in MK count from the prepulmonary to postpulmonary circulation. The fact that the latter was observed in the control groups but not in the hyperoxia groups made it unlikely that the lower lung MK count in the hyperoxia groups was due to more complete MK fragmentation. An alternative and probably more plausible speculation is that there was an inability of the damaged pulmonary capillary vessels in retaining circulating MK, which might contribute to the dysfunction of the pulmonary capillary bed in releasing platelets from MK.

In addition to impaired platelet release from MK, evidence of pulmonary platelet consumption was also present in our 

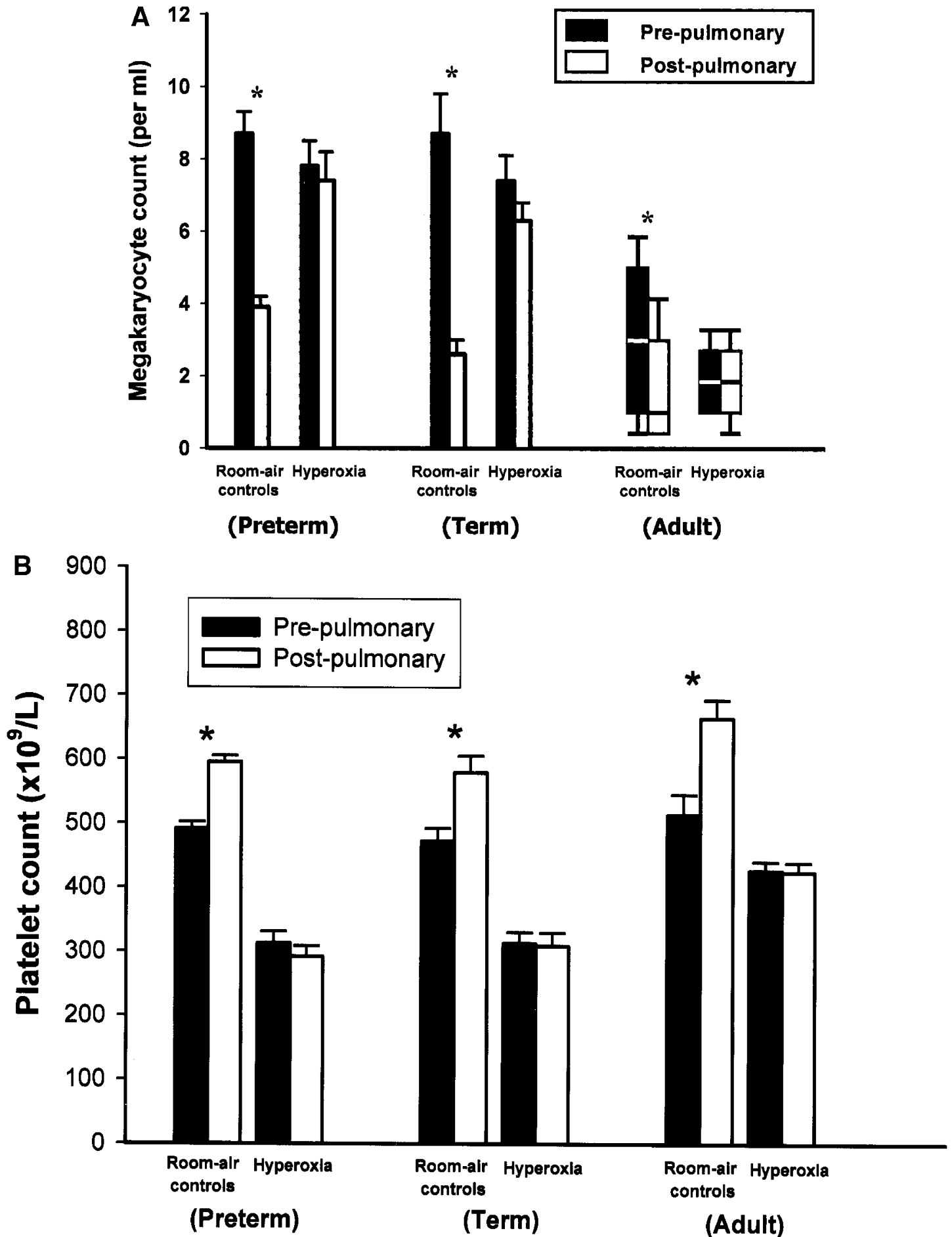

Figure 5. Megakaryocyte (MK) and platelet counts in the prepulmonary and postpulmonary blood of the animals with and without hyperoxic lung damage. In the preterm neonatal, term neonatal, and adult $(n=10,10$, and 18, respectively, in each of hyperoxia and control groups) rats, the postpulmonary MK count $(A)$ was significantly lower than the prepulmonary count $\left({ }^{*} p<0.001\right)$ in the room-air controls but not in those with hyperoxic lung damage. In contrast, the platelet counts $(B)$ was significantly higher in the postpulmonary blood than in the prepulmonary blood in the room-air controls $(* p<0.001)$. Again no difference in platelet count was observed between the prepulmonary and postpulmonary blood in those with hyperoxic lung damage. Values are expressed as mean (Sem) except for the adult prepulmonary and postpulmonary MK counts, which are not normally distributed and are therefore expressed as median (interquartile range).

animals with hyperoxic lung injury. In the neonatal term and preterm rats, exposure to hyperoxia for $7 \mathrm{~d}$ resulted in patchy areas of gross and histologic lung damage with extensive thrombi formation. The adult rats were exposed to hyperoxia for a much briefer period of $60 \mathrm{~h}$ because of their intolerance to hyperoxia and high mortality after $72 \mathrm{~h}$ of hyperoxic exposure (30). Although thrombi formation was not detected in their lungs under light microscopy, electron microscopy revealed extensive platelet aggregation and platelet adhesion to the endothelium. 


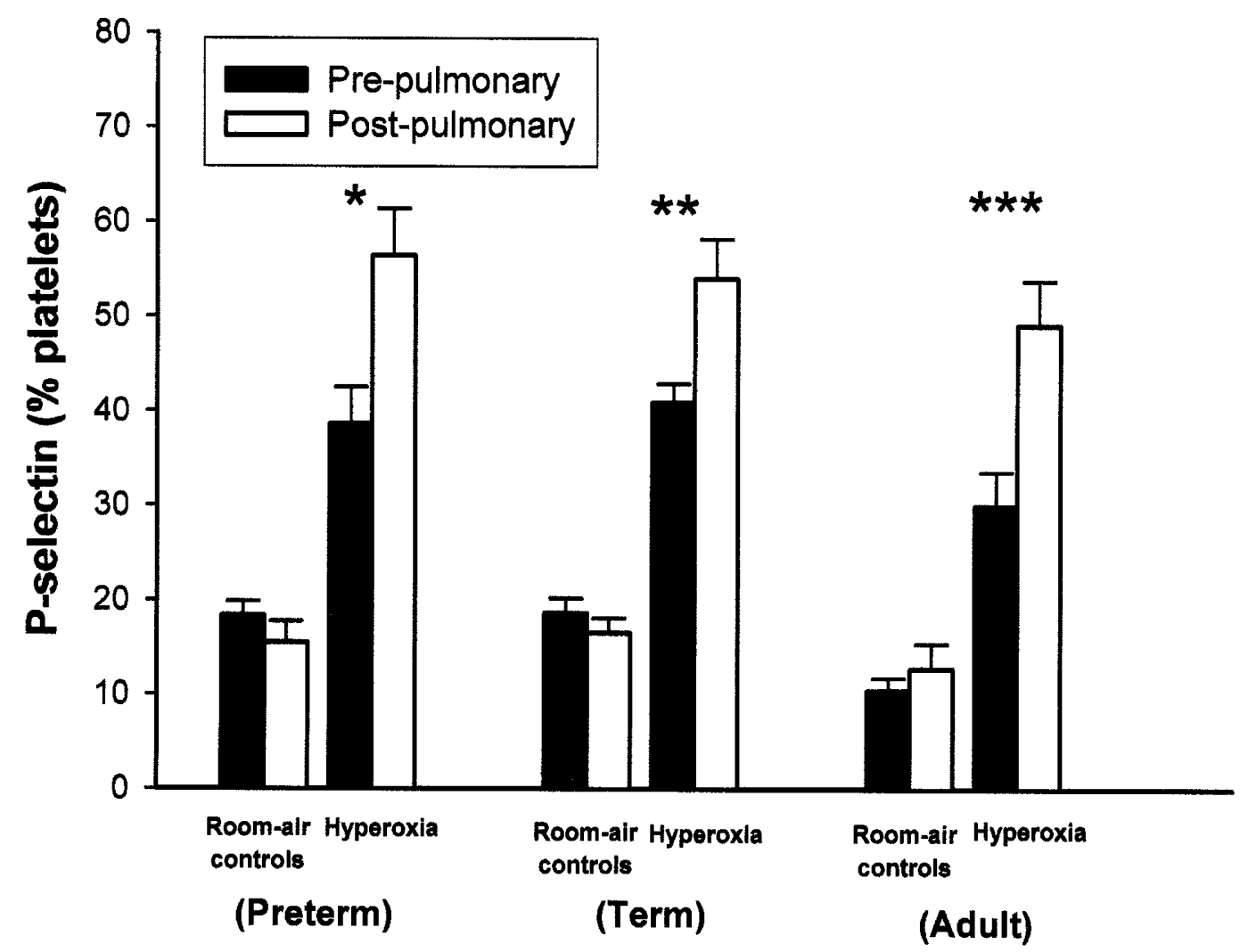

Figure 6. P-selectin expression in the prepulmonary and postpulmonary blood of the animals with and without hyperoxic lung damage. In the preterm neonatal, term neonatal, and adult ( $n=10,10$, and 18, respectively, in each of hyperoxia and control groups) rats, the postpulmonary blood P-selectin expression was significantly higher than that in the prepulmonary blood in the animals with hyperoxic lung damage $(* p=0.02, * * p=0.002, * * * p=0.003)$. There was no difference between the prepulmonary and postpulmonary P-selectin levels in the room-air controls. Comparing the baseline (prepulmonary) values between the lung-damaged and control rats, P-selectin levels were significantly higher in the lung-damaged group $(p<0.001$ in all three groups). Values are expressed as mean (SEM).

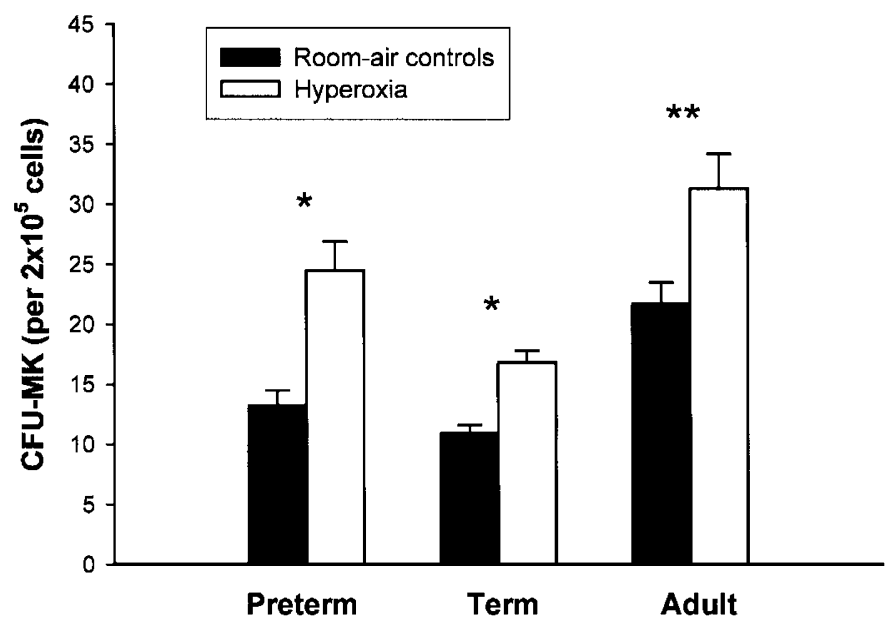

Figure 7. Megakaryocyte colony forming units (CFU-MK) in the bone marrow of the preterm neonatal, term neonatal, and adult $(n=26,30$, and 18 , respectively, in each of hyperoxia and control groups) rats with and without hyperoxic lung damage. In all three groups, the CFU-MK count was significantly higher in the hyperoxia lung-damaged rats than in their respective controls. Values are expressed as mean (SEM). $\left({ }^{*} p<0.001,{ }^{* *} p=0.008\right)$

By estimating the prepulmonary and postpulmonary Pselectin level in the animals, our findings also provided evidence of platelet activation by the injured lung. P-selectin is an adhesion receptor present in the platelet $\alpha$-granules mem- branes. Platelet activation is followed by its rapid expression on the platelet surface (36). The activated platelets have shortened survival time (37), and play an active role in intravascular platelet aggregation and thrombus formation presumably as a result of their increased surface adhesiveness $(38,39)$. Increase in circulating activated platelets has been associated with trauma of the vascular endothelium (40) and adult respiratory distress syndrome (41). Compared with the room-air controls, both adult and neonatal (preterm and term) rats with hyperoxic lung damage had significantly higher baseline (prepulmonary) P-selectin levels. Passage of the platelets through the lung significantly increased their P-selectin expression in the lungdamaged animals but not the controls, confirming that the damaged lung was the site of platelet activation. The relationship among hyperoxia, lung injury, and platelet activation is complex and not well understood. Hyperoxic lung damage and the resultant inflammatory reactions might lead to platelet activation possibly through the action of cytokines such as tumor-necrosis factor $(42,43)$. Platelet activation might also be a direct effect of hyperoxic exposure, which had been observed to directly induce expression of surface adhesion molecules on platelets, and sequestration of platelets in the lung (38). Irrespective of the mechanism of platelet activation, the activated platelets that are adherent to the endothelial surface might play an important role in lung damage by binding circulating neu- 
trophils through a P-selectin mediated mechanism. These platelet-neutrophil interactions could be important in perpetuating inflammatory damage of the lung by recruiting and localizing neutrophils to the sites of vascular thrombosis (44).

Our findings thus demonstrated that in both adult and neonatal (term and preterm) rats, lung injury might affect platelet homeostasis by a number of possible mechanisms. The damaged lung had an impaired function as a manufacturer of platelets due to a reduction in platelet release from MK fragmentation. There was also an increase in platelet consumption due to platelet aggregation and thrombi formation in the damaged pulmonary capillary bed. As a result, there was no net increase in the platelet count in the circulating blood after its passage through the lung. This, together with the shortened survival of the activated platelets, led to a reduction in the peripheral platelet count. The significance of this reduction in platelet count was examined by studying its effect on megakaryocyte production in the bone marrow. A decrease in circulating platelets triggers off a feedback control mechanism, which increases the production of thrombopoietic cytokines $(44-49)$ that stimulate MK production in the bone marrow (50-53). In both the neonatal and adult rats with hyperoxic lung injury, the proliferation of MK progenitor cells, expressed as CFU-MK formation, was significantly greater than that in their respective controls, confirming that the magnitude of reduction in circulating platelets was of physiologic significance.

Unexplained reduction in platelet count is a common observation in newborns with lung diseases $(3,5,8,9)$. Our findings demonstrated for the first time that the condition might be caused by impaired platelet production and increased platelet consumption in the lung. It is a common practice in neonatal intensive care units to consider thrombocytopenia a sign of sepsis, and the affected infants are often subjected to a series of investigations and treatment for the alleged infection. A septic cause is however not identified in many of these infants and the thrombocytopenia persists despite a full course of antibiotic treatment. While sepsis should remain an important consideration in any infant with reduction in platelet count, our findings provide an alternative explanation for this perplexing phenomenon.

Acknowledgments. We would like to thank Mr. Eric Wong, statistician, Center of Clinical Trial and Epidemiologic Research, Medical Faculty, The Chinese University of Hong Kong for his statistical assistance, and Mr. Raymond Wong, medical technologist, The Department of Paediatrics, The Chinese University of Hong Kong for his assistance in the assay of P-selectin expression.

\section{REFERENCES}

1. Burrows RF, Kelton JG 1990 Thrombocytopenia at delivery: a prospective survey of 6715 deliveries. Am J Obstet Gynecol 162:731-734

2. Burrows RF, Kelton JG 1988 Incidentally detected thrombocytopenia in healthy mothers and their infants. N Engl J Med 319:142-145

3. Castle V, Andrew M, Kelton J, Giron D, Johnston M, Carter C 1986 Frequency and mechanism of neonatal thrombocytopenia. J Pediatr 108(Pt 1):749-755

4. Murray NA, Roberts IA 1996 Circulating megakaryocytes and their progenitors in early thrombocytopenia in preterm neonates. Pediatr Res 40:112-119

5. Mehta P, Vasa R, Neumann L, Karpatkin M 1980 Thrombocytopenia in the high-risk infant. J Pediatr 97:791-794

6. Castle V, Coates G, Kelton JG, Andrew M 1987 111In-oxine platelet survivals in thrombocytopenic infants. Blood 70:652-656
7. Zipursky A, Palko J, Milner R, Akenzua GI 1976 The hematology of bacterial infections in premature infants. Pediatrics 57:839-853

8. Ballin A, Koren G, Kohelet D, Burger R, Greenwald M, Bryan AC, Zipursky A 1987 Reduction of platelet counts induced by mechanical ventilation in newborn infants. J Pediatr 111:445-449

9. Kohelet D, Perlman M, Hanna G, Ballin A 1990 Reduced platelet counts in neonatal respiratory distress syndrome. Biol Neonate 57:334-342

10. Schmidt B, Vegh P, Weitz J, Johnston M, Caco C, Roberts R 1992 Thrombin/ antithrombin III complex formation in the neonatal respiratory distress syndrome. Am Rev Respir Dis 145(Pt 1):767-770

11. Brus F, van Oeveren W, Okken A, Oetomo SB 1997 Number and activation of circulating polymorphonuclear leukocytes and platelets are associated with neonatal respiratory distress syndrome severity. Pediatrics 99:672-680

12. Steele P, Ellis JH, Jr., Weily HS, Genton E 1977 Platelet survival time in patients with hypoxemia and pulmonary hypertension. Circulation 55:660-661

13. Zucker-Franklin D, Philipp CS 2000 Platelet production in the pulmonary capillary bed: new ultrastructural evidence for an old concept. Am J Pathol 157:69-74

14. Levine RF, Eldor A, Shoff PK, Kirwin S, Tenza D 1993 Cramer EM. Circulating megakaryocytes: delivery of large numbers of intact, mature megakaryocytes to the lungs. Eur J Haematol 51:233-246

15. Graeve JLA, de Alarcon PA 1989 Megakaryocytopoiesis in the human fetus. Arch Dis Child 64:481-484

16. Woods MJ, Landon CR, Wagner BE, Greaves M, Trowbridge EA 1992 Isolation of circulating megakaryocytes in man. Med Lab Sci 49:252-258

17. Martin JF, Slater DN, Trowbridge EA 1983 Abnormal intrapulmonary platelet production: a possible cause of vascular and lung disease. Lancet 1(8328):793-796

18. Pedersen NT 1971 Circulating megakaryocytes in blood from the inferior vena cava in adult rats. Scand J Haematol 8:223-230

19. Pedersen NT 1974 The pulmonary vessels as a filter for circulating megakaryocytes in rats. Scand J Haematol 13:225-231

20. Aschoff L 1893 Üeber capilläre Embolie von riesenkernhaltigen Zellen. Virchows Arch Pathol Anat Physiol Klin Med 134:11-26

21. Howell WH, Donohue DD 1937 The production of blood platelets in the lungs. J Exp Med 65:171-204

22. Kaufman RM, Airo R, Pollack S, Crosby WH 1965 Circulating megakaryocytes and platelets release in the lung. Blood 26:720-731

23. Kallinikos-Maniakas A 1969 Megakaryocytes and platelets in central venous and arterial blood. Acta Haematol 42:330-335

24. Pedersen NT 1978 Occurrence of megakaryocytes in various vessels and their retention in the pulmonary capillaries in man. Scand J Haematol 21:369-375

25. Wilde NT, Burgess R, Keenan DJ, Lucas GS 1997 The effect of cardiopulmonary bypass on circulating megakaryocytes. Br J Haematol 98:322-327

26. Trowbridge EA, Martin JF, Slater DN 1982 Evidence for a theory of physical fragmentation of megakaryocytes, implying that all platelets are produced in the pulmonary circulation. Thromb Res 28:461-475

27. Tanswell AK, Wong L, Possmayer F, Freeman BA 1989 The preterm rat: a model for studies of acute and chronic neonatal lung disease. Pediatr Res 25:525-529

28. Deng H, Mason SN, Auten RL, Jr 2000 Lung inflammation in hyperoxia can be prevented by antichemokine treatment in newborn rats. Am J Respir Crit Care Med $162: 2316-2323$

29. Appleby CJ, Towner RA 2001 Magnetic resonance imaging of pulmonary damage in the term and premature rat neonate exposed to hyperoxia. Pediatr Res 50:502-507

30. Iwata M, Takagi K, Satake T, Sugiyama S, Ozawa T 1986 Mechanism of oxygen toxicity in rat lungs. Lung 164:93-106

31. Chen Y, Martinez MA, Frank L 1997 Prenatal dexamethasone administration to premature rats exposed to prolonged hyperoxia: a new rat model of pulmonary fibrosis (bronchopulmonary dysplasia). J Pediatr 130:409-416

32. Yang M, Chesterman CN, Chong BH 1995 Recombinant PDGF enhances megakaryocytopoiesis in vitro. Br J Haematol 91:285-289

33. Woods MJ, Greaves M, Smith GH, Trowbridge EA 1993 The fate of circulating megakaryocytes during cardiopulmonary bypass. J Thorac Cardiovasc Surg 106:658-663

34. Shattil SJ, Cunningham M, Hoxie JA 1987 Detection of activated platelets in whole blood using activation- dependent monoclonal antibodies and flow cytometry. Blood 70:307-315

35. Han ZC, Briere J, Abgrall JF, Sensebe L, Nedellec G, Parent D, Guern G 1987 Spontaneous formation of megakaryocyte progenitors (CFU-MK) in primary thrombocythaemia. Acta Haematol 78:51-53

36. Berman CL, Yeo EL, Wencel-Drake JD, Furie BC, Ginsberg MH, Furie B 1986 A platelet alpha granule membrane protein that is associated with the plasma membrane after activation. Characterization and subcellular localization of platelet activationdependent granule-external membrane protein. J Clin Invest 78:130-137

37. Doyle DJ, Chesterman CN, Cade JF, McGready JR, Rennie GC, Morgan FJ 1980 Plasma concentrations of platelet-specific proteins correlated with platelet survival. Blood 55:82-84

38. Barazzone C, Tacchini-Cottier F, Vesin C, Rochat AF, Piguet PF 1996 Hyperoxia induces platelet activation and lung sequestration: an event dependent on tumor necrosis factor-alpha and CD11a. Am J Respir Cell Mol Biol 15:107-114

39. Baumgartner HR, Hosang M 1988 Platelets, platelet-derived growth factor and arteriosclerosis. Experientia 44:109-112

40. Chignier E, Parise M, McGregor L, Delabre C, Faucompret S, McGregor J 1994 A P-selectin/CD62P monoclonal antibody (LYP-20), in tandem with flow cytometry, detects in vivo activated circulating rat platelets in severe vascular trauma. Thromb Haemost 72:745-749

41. George JN, Pickett EB, Saucerman S, McEver RP, Kunicki TJ, Kieffer N, Newman PJ 1986 Platelet surface glycoproteins. Studies on resting and activated platelets and platelet 
membrane microparticles in normal subjects, and observations in patients during adult respiratory distress syndrome and cardiac surgery. J Clin Invest 78:340-348

42. Welty SE, Rivera JL, Elliston JF, Smith CV, Zeb T, Ballantyne CM, Montgomery CA, Hansen TN 1993 Increases in lung tissue expression of intercellular adhesion molecule- 1 are associated with hyperoxic lung injury and inflammation in mice. Am J Respir Cell Mol Biol 9:393-400

43. Jensen JC, Pogrebniak HW, Pass HI, Buresh C, Merino MJ, Kauffman D, Venzon D Langstein HN, Norton JA 1992 . Role of tumor necrosis factor in oxygen toxicity. J Appl Physiol 72:1902-1907

44. Dore M 1998 Platelet-leukocyte interactions. Am Heart J 135(Pt 2 Suppl):S146-S151

45. Kaushansky K 1995 Thrombopoietin: the primary regulator of megakaryocyte and platelet production. Thromb Haemost 74:521-525

46. Ishibashi T, Kimura H, Shikama Y, Uchida T, Kariyone S, Hirano T, Kishimoto T, Takatsuki F, Akiyama Y 1989 Interleukin-6 is a potent thrombopoietic factor in vivo in mice. Blood 74:1241-1244

47. Ishibashi T, Kimura H, Uchida T, Kariyone S, Friese P, Burstein SA 1989 Human interleukin 6 is a direct promoter of maturation of megakaryocytes in vitro. Proc Nat Acad Sci USA 86:5953-5957
48. Brandt E, Ernst M, Loppnow H, Flad HD 1989 Characterization of a platelet derived factor modulating phagocyte functions and cooperating with interleukin 1. Lymphokine Res 8:281-287

49. Yang M, Li K, Chui CM, Yuen PM, Chan PK, Chuen CK, Li CK, Fok TF 2000 Expression of interleukin (IL) 1 type I and type II receptors in megakaryocytic cells and enhancing effects of IL-1beta on megakaryocytopoiesis and NF-E2 expression. Br J Haematol 111:371-380

50. Carrington PA, Hill RJ, Stenberg PE, Levin J, Corash L, Schreurs J, Baker G, Levin FC 1991 Multiple in vivo effects of interleukin-3 and interleukin-6 on murine megakaryocytopoiesis. Blood 77:34-41

51. Chang M, Suen Y, Meng G, Buzby JS, Bussel J, Shen V, van de V, Cairo MS 1996 Differential mechanisms in the regulation of endogenous levels of thrombopoietin and interleukin-11 during thrombocytopenia: insight into the regulation of platelet production. Blood 88:3354-3362

52. Williams N, Bertoncello I, Jackson H, Arnold J, Kavnoudias H 1992 The role of interleukin 6 in megakaryocyte formation, megakaryocyte development and platelet production. Ciba Found Symp 167:160-170

53. Long MW, Henry RL 1979 Thrombocytosis-induced suppression of small acetylcholinesterase-positive cells in bone marrow of rats. Blood 54:1338 